\title{
Vulnerabilidad a la autolesión en el autismo
}

\author{
Isabel Paula-Pérez, Josep Artigas-Pallarés
}

Introducción. El porqué de la autolesión en las personas con autismo no parece tener una explicación simple, sencilla y única. La predisposición biológica, determinados estados psicológicos de estrés, un procesamiento sensorial atípico, alteraciones en la comunicación, problemas médicos, limitaciones en la regulación emocional, entre otros, pueden llevan a la persona con autismo a dañarse.

Objetivo. En este artículo se diferencia, en primer lugar, la autolesión relacionada con alteraciones del neurodesarrollo, como el autismo y la autolesión vinculada a otras condiciones psiquiátricas; y en segundo lugar, se propone una primera aproximación a un modelo integral de comprensión de la autolesión en el autismo.

Desarrollo. Algunas de las hipótesis explicativas de la autolesión en el autismo están centradas en el procesamiento sensorial atípico, en alteraciones de la comunicación y problemas médicos, y en la desregulación emocional.

Conclusiones. La limitada investigación al respecto y la confusión de los datos que se arrojan ha generado un especial esfuerzo por separar los hechos de las suposiciones en todo lo relativo a este tema. Esta modesta propuesta inicial permite elaborar una hoja de ruta que oriente y ayude a las personas con autismo, a sus familias y a los profesionales en el proceso de disminuir o eliminar este comportamiento. Se sugiere dejar de hablar de la autolesión como comportamiento disruptivo y más bien interpretarla como una (inadecuada) respuesta autorregulatoria al estrés.

Palabras clave. Alteraciones de la comunicación. Autismo. Autolesión. Desregulación emocional. Problemas médicos. Procesamiento sensorial.

\section{Introducción}

¿Por qué buscar el daño que todos evitamos? Parece inconcebible que lo que la mayoría de las personas temen y evitan -el dolor- sea buscado por algunas personas. No lesionarnos, no dañarnos es una de las motivaciones biológicas más básicas y, a pesar de ello, aproximadamente un 50\% de las personas con autismo se hiere de una manera u otra aunque sólo sea en algún período de su ciclo vital $[1,2]$. Una parte significativa de ellos (aproximadamente un 14\%) lo hace de manera repetida y con un nivel de intensidad alto.

Las razones para analizar y recopilar evidencias sobre este tema son claras y contundentes:

- Los graves problemas de salud que las autolesiones pueden provocar, como fracturas, infecciones recurrentes, malformaciones físicas o desprendimiento de retina/ceguera, entre otros [3].

- Su alta tendencia a cronificarse, ya que comportamientos autolesivos que pueden parecer menos graves en la infancia pueden ser potencialmente muy dañinos [4].

- El impacto que tiene en la calidad de vida de la persona que lo padece, ya que la aísla y además limita en gran medida su acceso a actividades en la comunidad y el desarrollo de las relaciones sociales [5].

- La angustia significativa que estos comportamientos generan en los miembros de la familia. Los padres cuyos hijos muestran un alto nivel de autolesión manifiestan mayor deterioro de su calidad de vida en comparación con padres cuyos hijos tienen un nivel bajo o nulo de autolesión [6,7].

A pesar de las casi cinco décadas de investigación en la temática, hay pocas evidencias de que las conclusiones más sólidas se hayan traducido en intervenciones eficaces. En este artículo se propone una primera aproximación a un modelo integral de comprensión de la autolesión en el autismo basado en evidencias que permita la identificación de objetivos para la evaluación clínica y una intervención más adecuada.

\section{Autolesión en el autismo frente a autolesión en otras condiciones psiquiátricas}

El concepto de autolesión se ha utilizado de manera confusa por investigadores y profesionales para describir fenómenos muy diferentes. ¿Qué tienen que
Departamento de Métodos de Investigación y Diagnóstico en Educación, MIDE; Universitat de Barcelona; Barcelona (I. PaulaPérez). Centre Mèdic Psyncron; Sabadell, Barcelona, España (J. Artigas-Pallarés).

Correspondencia:

Dra. Isabel Paula Pérez. Campus Mundet. Departamento MIDE. Universitat de Barcelona. Edif. Llevant, 2. . piso, despacho 264. E-08035 Barcelona.

E-mail:

isabelpaula@ub.edu

Declaración de intereses: Los autores manifiestan la inexistencia de conflictos de interés en relación con este artículo.

Aceptado tras revisión externa: 20.01.16.

Cómo citar este artículo: Paula-Pérez I, Artigas-Pallarés J. Vulnerabilidad a la autolesión en el autismo. Rev Neurol 2016; 62 (Supl 1): S27-32.

(c) 2016 Revista de Neurología 
ver los cortes en la piel con una hoja de afeitar o las quemaduras autoprovocadas con una colilla con los golpes en la cabeza o con las mordeduras en las manos que se provoca la persona con autismo de manera repetitiva y casi estereotipada? La respuesta es compleja. El Manual diagnóstico y estadístico de los trastornos mentales, quinta edición [8], abre por primera vez el debate sobre la autolesión en un apartado dedicado a 'afecciones que necesitan más estudio', y en él se especifica la 'autolesión no suicida' (nonsuicidal self-injury). ¿De qué hablamos cuando hablamos de la autolesión en trastornos psiquiátricos distintos del autismo? Se contempla una frecuencia de, al menos, cinco días en el último año en los que la persona se inflige, intencionadamente, lesiones en la superficie corporal que producen sangrado, hematomas o dolor (por ejemplo, cortarse, quemarse, pincharse, golpearse, frotarse la piel en exceso), con la expectativa de que la lesión sólo conlleve un daño físico leve o moderado. Además, la persona realiza la conducta autolesiva con una o más de las siguientes expectativas: para aliviar un sentimiento o estado cognitivo negativo, para resolver una dificultad interpersonal o para inducir un estado de sentimientos positivos.

Para acercarnos a la cuestión de qué hablamos cuando hablamos de la autolesión en el autismo, debemos recurrir a la investigación con esta población [9]. Algunas de las particularidades que caracterizan la autolesión en el autismo son:

- El comportamiento provoca daño físico, más comúnmente en forma de daño a los tejidos corporales (moratones, arañazos, mordeduras, usualmente en la cara, la cabeza y las extremidades), en contraposición con el envenenamiento, las quemaduras o los cortes.

- El comportamiento se basa, fundamentalmente, en un movimiento rítmico, repetitivo y constante con el que pueden provocarse varios golpes por minuto; por ejemplo, golpearse con la mano en la cabeza una y otra vez.

- La autolesión no está predeterminada, la persona no la idea ni la planifica.

- Puede producirse una explosión después de largos períodos sin autolesiones.

- A menudo se llevan a cabo en aparente ausencia de dolor.

- Cuanto más grave es la discapacidad intelectual o la gravedad del autismo, más probable es que la persona evidencie comportamientos autolesivos.

- La frustración, la ansiedad y el deseo concomitante de escapar de una situación pueden formar parte de los motivos que desencadenan la autolesión.
La topografía de la autolesión en otros trastornos psiquiátricos diferentes del autismo suele ser áreas del cuerpo asociadas con muy baja letalidad, como los antebrazos y la parte superior de las piernas [10]. Cortarse es el método más comúnmente utilizado [11] y suele hacerse uso de instrumentos como cuchillos, agujas, cuchillas u otros objetos afilados; colillas encendidas o gomas de borrar para frotarse la piel, provocando a menudo sangrado y dejando un patrón de cicatrices característico [12]. Además, a diferencia del autismo, en estos casos se suele aprender por recomendación u observación de otra persona [8]. La topografía de la autolesión en el autismo es heterogénea sin hacer uso de instrumentos: darse golpes en la cabeza, la cara, la mandíbula o los ojos, morderse, tirarse de las uñas, arañarse, tirarse del pelo o de los dientes, pellizcarse, darse puñetazos, darse bofetadas en el oído o en la cabe$\mathrm{za}, \mathrm{o}$ darse con la rodilla en la cara, entre otros [13]. Otros menos frecuentes son ingerir aire, meterse el dedo en aberturas como los ojos, la nariz o los oídos, tomar demasiado líquido o agitar la cabeza enérgicamente, entre otros.

\section{Posibles hipótesis explicativas de la autolesión en el autismo basadas en evidencias}

El porqué de la autolesión en las personas con autismo no parece tener una explicación simple, sencilla y única. ¿Qué experiencias, predisposiciones y estados psicológicos llevan a la persona con autismo a dañarse? ¿Factores biológicos, psicológicos, sociales? En estos momentos contamos más con suposiciones ('creo que...') que con evidencias científicas, por lo que, a menudo, la demanda de ayuda de los padres a los profesionales se percibe con inseguridad por parte de éstos. Estas páginas son un intento por comenzar a sistematizar algunas evidencias.

\section{Procesamiento sensorial atípico}

La primera hipótesis sostiene que los niños con autismo que se autolesionan pueden ser menos sensibles a la estimulación dolorosa. El procesamiento somatosensorial atípico se asocia con una posible hiperactividad del sistema opioidérgico. Con la autolesión, la persona libera y aumenta la producción de endorfinas, provocando la analgesia y la reducción de la percepción de los estímulos sensoriales [14], entre ellos, el dolor. Se provoca un efecto anestésico a corto plazo, de manera que la persona deja de sentir dolor al autolesionarse [15]. Esta conclusión se basa en investigaciones sobre los antagonis- 
tas de los opiáceos para el tratamiento de la autolesión en algunas personas con autismo [16]. El antagonista más conocido de los receptores opioides OPRM1, OPRD1 y OPRK1 es la naltrexona (un inhibidor de las endorfinas), que puede disminuir la autolesión al bloquear la respuesta de placer [17]. La hipótesis sostiene que esta sensación de placer refuerza el comportamiento autolesivo y da lugar a un aumento de la probabilidad de recurrencia.

La segunda hipótesis afirma que la autolesión aumenta la conciencia de la posición del cuerpo en el espacio producto de una pobre sensación propioceptiva; por ejemplo, dándose pellizcos en la piel, frotándose o rascándose hasta hacerse sangre. Una autolesión repetitiva puede aumentar la conciencia somatosensorial, por lo que la persona con autismo busca activamente esa estimulación propioceptiva y táctil. Dicha autoestimulación puede acabar convirtiéndose en autolesión. Esto se relacionaría también con la hiporreactividad a estímulos sensoriales que conduce a la persona con autismo a sentir la necesidad de buscar estimulación sensorial, especialmente en ausencia de factores desencadenantes ambientales. En cualquier caso, esta teoría se basaría en la hipótesis de que la autolesión es una manera extrema de procesamiento sensorial atípico [18], con la finalidad de alcanzar un grado más alto de estimulación.

Merecería la atención de un artículo aparte las evidencias científicas relativas al mecanismo de neurotransmisión y neuromodulación de la sensación dolorosa en las personas con autismo y, más especialmente, la integración de la respuesta al dolor en los centros superiores (estructuras encefálicas). Se mantiene la hipótesis de que la autolesión puede estar asociada con alteraciones en las regiones del cerebro que procesan los componentes sensoriales discriminativos y afectivos del dolor y pueden reflejar un procesamiento somatosensorial atípico comúnmente hallado en las personas con esta condición [19].

\section{Alteraciones de la comunicación y problemas médicos}

Los niños con autismo a menudo tienen dificultades para expresar sus pensamientos, deseos, necesidades o ideas. Queda bajo la responsabilidad de sus personas cercanas que anticipen lo que necesitan y que interpreten lo que quieren en un momento dado. Éste es, por razones obvias, un sistema imperfecto, ya que, a menudo, conduce a la frustración cuando sus necesidades no se satisfacen de manera adecuada. Son múltiples y coincidentes los estudios en los que se asocia la autolesión en el autismo con alteraciones funcionales de la comunica- ción lingüística. Una comunicación receptiva y expresiva deficitaria está ligada a niveles más altos de autolesión [20,21]. Un creciente cuerpo de investigación ha examinado el grado en que las personas se autolesionan como medio para comunicarse con los demás a través de la evaluación funcional de la autolesión. En el marco de la psicología conductista, se interpretan dos funciones básicas de la autolesión: el refuerzo social negativo, con el que la persona evita o escapa de una demanda interpersonal; y el refuerzo social positivo, con el que la autolesión ayuda a la persona a obtener la atención de los demás o el acceso a objetos y actividades [22].

Disponemos de evidencias indirectas que confirman que la persona con autismo también puede acceder a la autolesión cuando siente un malestar o dolor físico. Ante la pregunta ‘qué te duele?', los niños con autismo suelen tener dificultades para responder. Sentir un dolor agudo y no ser capaz de decir a alguien dónde o cuánto te duele puede ser emocionalmente muy frustrante. Hay dolores internos del cuerpo que son difíciles de explicar, por lo que los problemas de salud y el dolor asociado a éstos son una dificultad añadida para las personas con autismo, especialmente las no verbales. Al no poder comunicar ese malestar, éste puede pasar desapercibido y no tratarse, con lo que la vivencia de dolor que acompaña a la persona puede llegar a ser muy estresante.

El dolor asociado con dolores de cabeza o infecciones del oído medio puede ser un factor que contribuya, específicamente, a comportamientos como golpearse la cabeza con los puños y las manos o darse cabezazos [23]. Del mismo modo, el dolor ocular puede conducir a la presión en los ojos; una infección de muelas o una sinusitis pueden conducir a presionarse la cabeza, la cara o la mandíbula. Otra de esas hipótesis apunta a problemas gastrointestinales. El Dr. Timothy Buie, pediatra gastroenterólogo del Hospital General de Massachusetts, sugiere que, cuando los problemas gastrointestinales (reflujo gastroesofágico, esofagitis, etc.) están presentes en niños con autismo, pueden aparecer comportamientos no asociados típicamente con el reflujo gastroesofágico, incluyendo la agresión o la autolesión. Sugerimos que cualquier paciente que se autolesiona sea sometido a una evaluación médica completa [24].

No deberíamos interpretar por defecto la autolesión como un intento de la persona por manipularnos. Sería más apropiado considerarla un recurso para expresar que necesita ayuda. Obviamente, la enseñanza de sistemas aumentativos y alternativos de comunicación será, en estos casos, una estrate- 
Figura. Aproximación a un primer modelo integral de comprensión de la autolesión en el autismo.

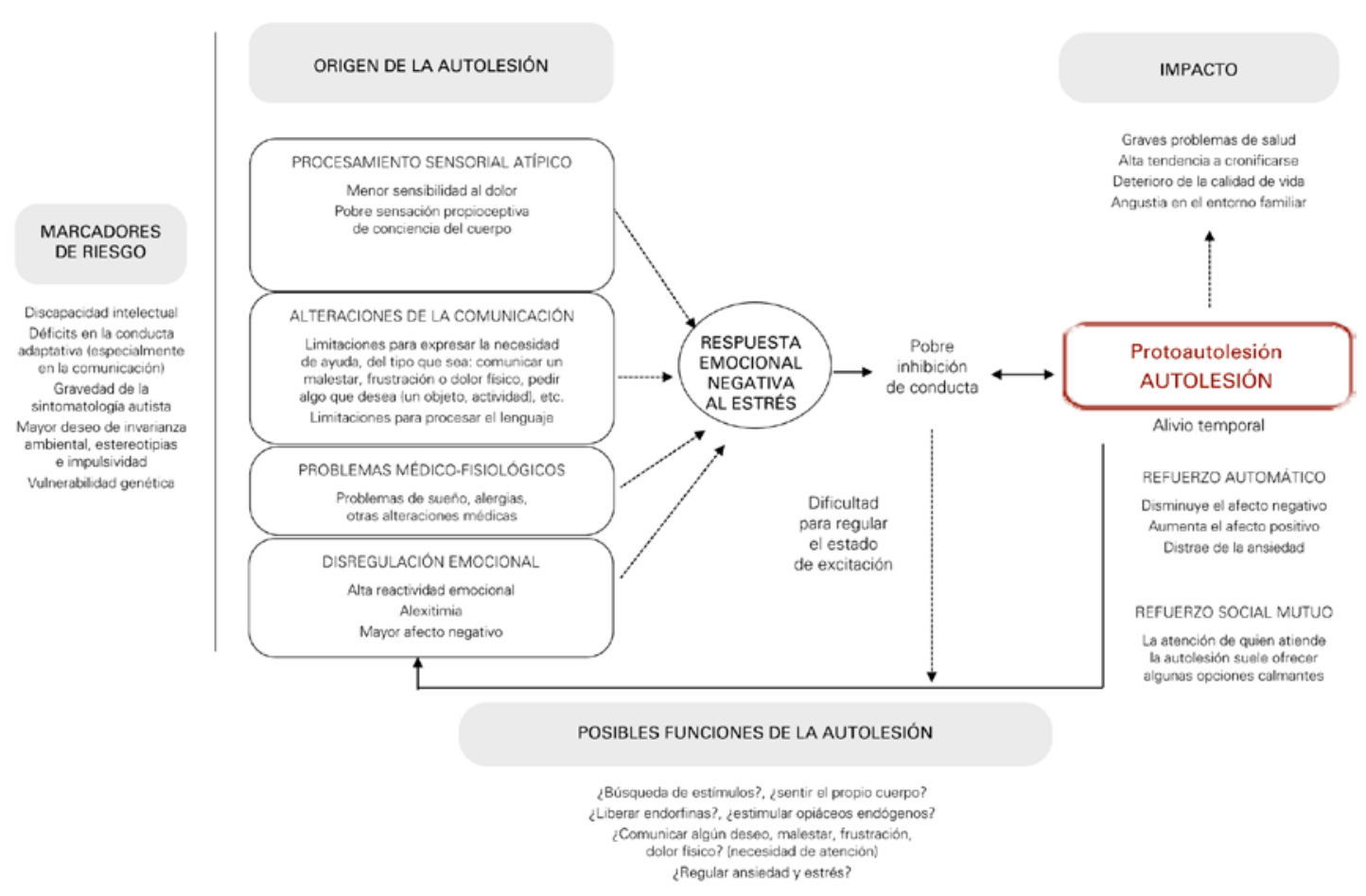

gia de afrontamiento prioritaria, pero en ningún caso la panacea, puesto que la autolesión puede producirse por otros motivos más allá de las alteraciones de la comunicación.

\section{Desregulación emocional}

Las evidencias clínicas sugieren que las personas con autismo tienen más problemas para regularse emocionalmente, lo que da como resultado no sólo un mayor afecto negativo, sino también el aumento de reacciones aparentemente desproporcionadas, normalmente llamadas crisis nerviosas (meltdown) [25]. En comparación con la población sin autismo, las personas con autismo utilizan 12 veces más servicios psiquiátricos relacionados con problemas de ansiedad y depresión, así como también problemas asociados con comportamientos externalizantes, como la autolesión y la agresión [26].

La hipótesis parte de la premisa de que las personas con autismo manifiestan una alta reactividad emocional; de hecho, la ansiedad es el trastorno comórbido más prevalente en las personas que manifiestan autolesión [27]. La ansiedad puede desempeñar un papel importante en el mantenimiento de las conductas autolesivas porque éstas se dan como respuesta refleja a altos niveles de activación generados por factores de estrés. A menudo sienten sobrecarga cognitiva o sensorial y 'explotan'. Después de desregularse, producto de su alta reactividad emocional, vuelven a su línea base de manera más lenta. Muchos de ellos encuentran en la autolesión un alivio para regularse emocionalmente.

Además, la alexitimia, presente de manera muy prevalente en el autismo [28], suele ser una característica común en las personas que se autolesionan: 'Creo que me autolesiono debido a que no puedo expresar mis emociones, no puedo nombrar mis sentimientos. Cuando me sobrecargo por esos sentimientos, me hago daño [...]. No te puedo decir qué sentimientos son, porque no sé cómo nombrarlos' [29]. La prospectiva de la investigación deberá abordar la relación entre alexitimia y autolesión, y la combinación de reactividad emocional alta y alta inexpresividad. Al ser personas menos propensas a reconocer estados de malestar y lo que les conduce a ellos, hacen un uso insuficiente de estrategias para pedir apoyo enfrascándose en comportamientos que denominados 'disruptivos', pero que en realidad son signos con los que transmiten que están angus- 
tiados, al mismo tiempo que actúan como mecanismos autorregulatorios para recuperar un cierto nivel de bienestar físico y emocional.

\section{Aproximación a un primer modelo integral de comprensión de la autolesión en el autismo}

Las hipótesis expuestas no son capaces de dar cuenta por sí solas del porqué de la autolesión en el autismo. Más bien, cada una de ellas representa una pieza de un rompecabezas complejo con factores de riesgo y circunstancias variadas que conducen a que la persona se autoinflija daño (Figura).

Se parte de una situación estimular que provoca una respuesta emocional negativa y desagradable que, en combinación con las dificultades de la persona para regularse en estado de excitación, la conducen a buscar maneras para evitar esa emoción negativa. La autolesión proporciona un alivio temporal a los sentimientos adversos. Después de hacerlo varias veces, se convierte en una respuesta automática, condicionada a la activación emocional desagradable. Pero esta hipótesis no explica por qué algunas personas con autismo se autolesionan y otras optan por poner en marcha otros comportamientos.

Además, 'el origen de la conducta no siempre es lo que sostiene el comportamiento' [30]. Independientemente del origen o la causa de la autolesión, hay que valorar qué es lo que mantiene el comportamiento autolesivo. ¿Aprende la persona con autismo que de esa manera consigue buscar la ayuda de las personas que le rodean? ¿Evita o escapa de situaciones que no le agradan, agobian o generan ansiedad? La reacción de alarma que crea en los demás proporciona una atención extraordinaria y ofrece algunas opciones calmantes que, a su vez, hacen que la persona siga recurriendo a ella para pedir ayuda y aliviar, nuevamente, el dolor. El matiz es casi imperceptible, pero importante: no es lo mismo querer llamar la atención que tener necesidad de buscar atención.

\section{Conclusiones}

Las personas con autismo son más vulnerables que otros colectivos de personas con trastornos del neurodesarrollo para manifestar comportamientos autolesivos. La intervención tendrá que ser coherente con la causa que provoca el comportamiento autolesivo: ¿reducir tensión?, ¿regular la ansiedad y el estrés?, ¿aliviar la sensación de vacío emocional (alexitimia)?, ¿comunicar dolor emocional?, ¿bus- car cuidado y apoyo?, ¿sentir el propio cuerpo?, ¿liberar endorfinas?, ¿es un ritual o un patrón de comportamiento? Algunas de las estrategias proactivas están basadas en la dieta sensorial (estrategias en los casos en los que la persona es menos sensible al dolor, o cuando la persona busca estimulación por problemas propioceptivos); en estrategias autorregulatorias de la ansiedad; en el aprendizaje de sistemas alternativos y aumentativos de comunicación; o en descartar cualquier tipo de problema médico. Algunas de las estrategias reactivas que habrá que valorar cuando la autolesión está fuertemente instaurada y el peligro físico para la persona es muy alto son los dispositivos de protección, los safety places y, en los casos en los que así se valore, la farmacología, entre otros, pero siempre bajo la constante supervisión de profesionales especializados y como último recurso.

En resumen, la autolesión debemos interpretarla como una (inadecuada) estrategia de afrontamiento para gestionar una variedad de estímulos y sentimientos negativos y abrumadores. Si la persona con autismo no puede comunicarlo, debemos ser nosotros quienes identifiquemos las razones por las que se autolesiona para generar, proponer y facilitar otras habilidades que resulten menos dañinas y más adaptativas. Sólo con una teoría integral basada en resultados empíricos podemos adaptar aún más nuestros esfuerzos de prevención y tratamiento. Debemos comprender que el nivel de angustia que siente alguien que se está haciendo daño a sí mismo es alto. La autolesión es, pues, un síntoma de un problema subyacente, por lo que si no detectamos y solucionamos el problema, dejar de autolesionarse no es un proceso fácil. La contención, física o farmacológica, no basta. 'Deshacerse del dolor sin dirigirse a la causa más profunda sería como apagar una alarma de incendios mientras aún hay fuego' [31].

\section{Bibliografía}

1. Bodfish JW, Symons FJ, Parker DE, Lewis MH. Varieties of repetitive behavior in autism: comparisons to mental retardation. J Autism Dev Disord 2000; 30: 237-43.

2. Baghdadli A, Pascal C, Grisi S, Aussilloux C. Risk factors for self-injurious behaviours among 222 young children with autistic disorders. J Intellect Disabil Res 2003; 47: 622-7.

3. Totsika V, Toogood S, Hastings RP, Lewis S. Persistence of challenging behaviours in adults with intellectual disability over a period of 11 years. J Intellect Disabil Res 2008; 52: 446-57.

4. Richman DM. Annotation: early intervention and prevention of self-injurious behaviour exhibited by young children with developmental disabilities. J Intellect Disabil Res 2008; 52: 3-17.

5. Chadwick O, Kusel Y, Cuddy M. Factors associated with the risk of behaviour problems in adolescents with severe intellectual disabilities. J Intellect Disabil Res 2008; 52: 864-76.

6. Rattaz C, Michelon C, Baghdadli A. Symptom severity as a risk factor for self-injurious behaviours in adolescents with 
autism spectrum disorders. J Intellect Disabil Res 2015; 59: 730-40.

7. Baghdadli A, Pry R, Michelon C, Rattaz C. Impact of autism in adolescents on parental quality of life. Qual Life Res 2014; 23: $1859-68$

8. American Psychiatric Association. Diagnostic and statistical manual of mental disorders, fifth edition (DSM-5). Arlington, VA: APA; 2013.

9. Matson JL, Turygin NC. How do researchers define self-injurious behavior? Res Dev Disabil 2012; 33: 1021-6.

10. Whitlock J, Muehlenkamp J, Purington A, Eckenrode J, Barreira P, Baral-Abrams G, et al. Nonsuicidal self-injury in a college population: general trends and sex differences. J Am Coll Health 2011; 59: 691-8.

11. Madge N, Hewitt A, Hawton K, De Wilde EJ, Corcoran P, Fekete $\mathrm{S}$, et al. Deliberate self-harm within an international community sample of young people: comparative findings from the Child \& Adolescent Self-harm in Europe (CASE) study. J Child Psychol Psychiatry 2008; 49: 667-77.

12. Klonsky ED, Muehlenkamp JJ. Self-injury: a research review for the practitioner. J Clin Psychol 2007; 63: 1045-56.

13. Lundqvist $O$. Psychometric properties and factor structure of the behavior problems inventory (BPI-01) in a Swedish community population of adults with intellectual disability. Res Dev Disabil 2011; 32: 2295-303.

14. Gillberg C. Endogenous opioids and opiate antagonists in autism: brief review of empirical findings and implications for clinicians. Dev Med Child Neurol 1995; 37: 239-45.

15. Sandman CA, Touchette P, Marion S, Lenjavi M, Chicz-Demet A. Disregulation of proopiomelanocortin and contagious maladaptive behavior. Regul Pept 2002; 108: 179-85.

16. Symons FJ, Thompson A, Rodriguez MC. Self-injurious behavior and the efficacy of naltrexone treatment: a quantitative synthesis. Ment Retard Dev Disabil Res Rev 2004; 10: 193-200.

17. Casner JA, Weinheimer B, Gualtieri CT. Naltrexone and self-injurious behavior: a retrospective population study. J Clin Psychopharmacol 1996; 16: 389-94.

18. Edelson SM. Implications of sensory stimulation in selfdestructive behavior. Am J Ment Defic 1984; 89: 140-5.
19. Tordjman S, Anderson GM, Botbol M, Brailly-Tabard S, Pérez-Díaz F, Graignic R, et al. Pain reactivity and plasma beta-endorphin in children and adolescents with autistic disorder. PLoS One 2009; 4: e5289.

20. Oliver C, Petty J, Ruddick L, Bacarese-Hamilton M. The association between repetitive, self-injurious and aggressive behavior in children with severe intellectual disability. J Autism Dev Disord 2012; 42: 910-9.

21. Matson JL, Wilkins J, Macken J. The relationship of challenging behaviors to severity and symptoms of autism spectrum disorders. J Ment Health Res Intellect Disabil 2008; 2: 29-44.

22. Nock MK, Prinstein MJ. Contextual features and behavioral functions of self-mutilation among adolescents. J Abnorm Psychol 2005; 114: 140-6.

23. Gualtieri CT. The differential diagnosis of self-injurious behavior in mentally retarded people. Psychopharmacol Bull 1988; 25: 358-63.

24. Buie T, Campbell DB, Fuchs GJ, Furuta GT, Levy J, Van de Water J, et al. Evaluation, diagnosis, and treatment of gastrointestinal disorders in individuals with ASDs: a consensus report. Pediatrics 2010; 125 (Suppl 1): S1-18.

25. Baker J. Positive strategies for managing and preventing out-of-control behavior: no more meltdowns. Arlington, VA: Future Horizons; 2008.

26. Prizant BM, Laurent A. Behavior is not the issue: an emotional regulation perspective on problem behavior. Part one of a part-two article. Autism Spectrum Quarterly 2011; 1: 29-30.

27. Moss S, Emerson E, Kiernan C, Turner S, Hatton C, Alborz A. Psychiatric symptoms in adults with learning disability and challenging behaviour. Br J Psychiatry 2000; 177: 452-6.

28. Pérez P, Martos JM, Llorente-Comí M. Alexitimia y síndrome de Asperger. Rev Neurol 2010; 50 (Supl 3): S85-90.

29. Taylor GJ, Bagby RM. Disorders of affect regulation: alexithymia in medical and psychiatric illness. New York: Cambridge University Press; 1997.

30. Weiss MJ, Wagner S, Goldberg S. Drawing the line: ten steps to constructive discipline-and achieving a great relationship with your kids. New York: Warner Books; 2006.

31. Burn S. Conversations with David Foster Wallace. Jackson, MS: University Press of Mississippi; 2012.

\section{Vulnerability to self-harm in autism}

Introduction. The reasons underlying self-harm in persons with autism do not appear to have a single, straightforward and simple explanation. Biological predisposition, certain psychological states involving stress, atypical sensory processing, communication disorders, medical problems, and limited emotional regulation, among others, can lead persons with autism to harm themselves.

Aims. In this article a distinction is drawn, first of all, between self-harm related to neurodevelopmental disorders, such as autism, and self-harm linked to other psychiatric conditions. Second, a preliminary approach to an integrated model for the understanding of self-harm in autism is proposed.

Development. Some of the hypotheses put forward to account for self-harm in autism are focused on atypical sensory processing, on communication disorders and medical problems, and on emotional dysregulation.

Conclusions. The limited number of studies conducted in this area and the inconsistency of the data resulting from them have led to great efforts being made to separate the facts from the suppositions in this subject. This modest initial proposal makes it possible to draw up a roadmap to guide and help persons with autism, their families and professionals in the process of reducing or eliminating this behaviour. It is suggested that self-harm should no longer be considered a disruptive behaviour and that it should be interpreted instead as an (inadequate) self-regulatory response to stress.

Key words. Autism. Communication disorders. Emotional dysregulation. Medical problems. Self-harm. Sensory processing. 